\title{
El caso de becas Maule (Chile), decisiones estratégicas: un enfoque desde la responsabilidad social universitaria para el logro de ventajas competitivas
}

Ivette M. Durán-Seguel ${ }^{1}$, Maximiliano E. Gallegos ${ }^{2}$, Francisco A. Ganga-Contreras ${ }^{3}$, y César A. Ahumada ${ }^{1}$

(1) Facultad de Ciencias Sociales y Económicas, Departamento de Economía y Administración, Universidad Católica del Maule. Avda San Miguel 3605, Talca, Chile (correo-e: iduran@ucm.cl; cahumada@ucm.cl)

(2) Facultad de Economía y Negocios, Universidad de Talca, Talca, Chile. (correo-e: mgallegos@utalca.cl)

(3) Departamento de Educación de la Facultad de Educación y Humanidades de la Universidad de Tarapacá. José

Victorino Lastarria 26, Santiago, Chile. (correo-e: franciscoganga@academicos.uta.cl)

Recibido Feb. 4, 2021; Aceptado Abr. 1, 2021; Versión final Jun. 30, 2021, Publicado Dic. 2021

\begin{abstract}
Resumen
Este estudio analiza como las decisiones estratégicas generan ventajas competitivas a través del fortalecimiento de alianzas estratégicas y desde el enfoque de la responsabilidad social universitaria. Se aplica una metodología de casos y análisis documental. El caso de las Becas Maule se remonta al año 2012, cuando la Facultad de Medicina de la Universidad Católica del Maule (UCM) (Chile) diseña un inédito programa de formación de 100 médicos especialistas en y para la región de Maule, con el objetivo de reducir la brecha paciente/médico. Los resultados muestran la importancia de: 1) la permanente conexión con el entorno para capitalizar oportunidades de forma temprana y 2) contar con información de calidad y oportuna para tomar decisiones que permitan dar respuesta a las necesidades de los más vulnerables de la región en temas de salud. Se concluye que la alineación estratégica al interior de la institución es un factor clave para el éxito del proceso de planeación e implementación del programa.
\end{abstract}

Palabras clave: decisiones estratégicas; ventajas competitivas; universidad; responsabilidad social

\section{The case of Maule (Chile) scholarships, strategic decisions: a university social responsibility approach to achieve competitive advantages}

\begin{abstract}
This study analyzes how strategic decisions develop competitive advantages by strengthening strategic alliances and by using a university social responsibility approach. The methodology applied includes a case study and a documentary research analysis. The Maule Scholarships started in the year 2012 when the Faculty of Medicine at the Universidad Católica del Maule (Chile) designed a medical specialty-training program for 100 doctors in the Maule province with the objective of reducing the gap between patients and doctors. The results show the importance of: 1) a permanent connection with the surroundings to capitalize on opportunities early on and 2) quality and timely information to make decisions that allow addressing the health issues of the most vulnerable people in the province. It is concluded that having a strategic alignment within the institution is a key factor for successfully planning and implementing the program.
\end{abstract}

Keywords: strategic decisions; competitive advantages; university; social responsibility 


\section{INTRODUCCIÓN}

Las decisiones estratégicas, con un enfoque de largo plazo, complejas, no rutinarias, desestructuradas, (Ganga-Contreras, et al, 2018) orientan de manera efectiva el rumbo de la organización y permiten el logro de ventajas competitivas (De Mesquita, et al., 2020). Facilitan de esta manera, la acción innovadora de dirección y liderazgo, permitiendo enfrentar problemas y oportunidades, así como cambios en el entorno de las organizaciones (Halibas, et al. 2017; Elbana, et al., 2020), además son fundamentales para las acciones organizativas y la competitividad a largo plazo (Calabretta, et al., 2017). Son estas decisiones estratégicas basadas en las definiciones institucionales como, por ejemplo, la misión de la organización, las encargadas de operacionalizarla.

Las universidades operacionalizan a través de las decisiones estratégicas la misión de la institución, relevando los ejes estratégicos que guían su quehacer (docencia, investigación, posgrado y vinculación con el medio), y donde se hace indispensable que no solo queden en declaraciones, especialmente aquellas acciones que impactan en el entorno de la universidad (Berghaeuser y Horlsche, 2019). Es por ello que se requiere un equilibrio, alineación estratégica, entre la estrategia y el contexto (Ghonim, et al., 2020). Las instituciones de educación superior, precisan aprovechar los recursos humanos y vínculos externos para mejorar la prestación de servicios y abordar las necesidades de la región (Barrios-Hernández y Olivero-Vega, 2020; Halibas, et al., 2017). En el último tiempo, han centrado su atención hacia el entorno social y hacia la gestión integral de la organización académica, planteando la responsabilidad social universitaria (RSU) como la capacidad que tiene la universidad de difundir y poner en práctica un conjunto de principios y valores generales y específicos, por medio de cuatro procesos claves, como son la gestión, docencia, investigación y la extensión universitaria, respondiendo así frente a la propia comunidad universitaria y el país donde está inserta (De Mesquita, et al., 2020), pero además, considerándola como una estrategia de gestión universitaria, que potencia iniciativas interdisciplinarias e interinstitucionales para dar respuesta a sus propios grupos de interés (Gaete, 2016; Larran y Andrades-Peña, 2015).

En este sentido, el rol de la universidad según, Martí y Martí-Vilar (2013) "es superior al de cualquier otra estructura pues en su haber está el ser o no ser, el dotar de principios y directrices bajo la atenta mirada de las sociedades, o sociedad global, en la cual ejerce su función" p.158. Sin embargo, y a pesar de la importancia que reviste el entorno para las universidades, los estudios que se realizan en el ámbito de las decisiones estratégicas que asumen las casas de estudio, aún no son suficientes. Lo anterior, queda de manifiesto, al analizar ámbitos diferentes a la innovación y/o tecnología, en los cuáles se ha enfocado la literatura (Halibas, et al., 2017; De Mesquita, 2020), y que consideran modelos que analizan la relación de la universidad con las empresas, dejando de lado experiencias de colaboración y alineación estratégica que tienen un gran impacto en la sociedad local y en el logro de ventajas competitivas para las propias universidades.

Es necesario entonces, fortalecer la alineación de las decisiones estratégicas y potenciarlas a través de alianzas estratégicas para lograr ventajas competitivas. Sin embargo, lo anterior no basta en el caso de una universidad pública, cuya misión la interpela a que dichas ventajas, no estén exentas del enfoque de responsabilidad social que se han impuesto muchas veces como un valor transversal a la institución. Para constatar lo anteriormente expuesto, se toma como ejemplo el caso de las Becas Maule, un programa de Becas de especialidad, para alumnos y exalumnos de la carrera de Medicina de la Universidad Católica del Maule, principalmente, y que se planificó, articuló e implementó de tal forma que llegó a generar una ventaja competitiva.

\section{OTROS ANTECEDENTES}

Se presenta una revisión crítica de los aspectos teóricos que han servido como marco de referencia para esta investigación. En la figura 1, se muestran en forma esquematizada los elementos teóricos a analizar, estos son: decisiones estratégicas, generación de ventajas competitivas como resultado de la dirección estratégica y responsabilidad social universitaria.

\section{Decisiones estratégicas}

Una decisión estratégica consiste en la determinación de los objetivos y metas a largo plazo, la adopción de los cursos de acción y la asignación de los recursos necesarios para alcanzar las metas establecidas por una organización, todo lo anterior enmarcado en un contexto (Elbana, et al., 2020, Calabretta, et al., 2017), por lo tanto, es una tarea esencial para la alta dirección, ya que permite a las organizaciones alinear sus recursos y capacidades con las amenazas y las oportunidades que existen en el medio ambiente (Rodríguez-Cruz y Pinto, 2018). 


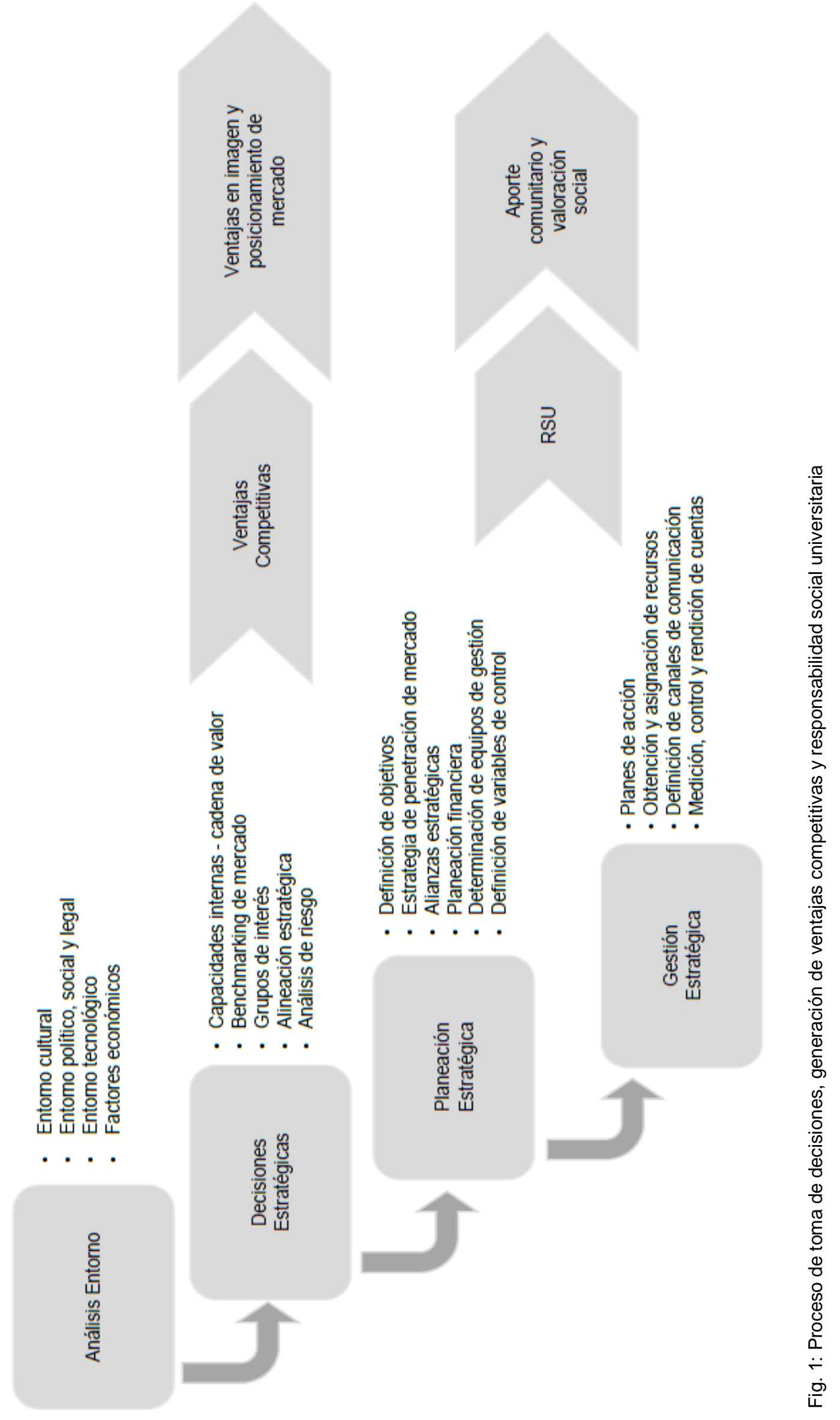


Las decisiones estratégicas permiten implementar el rumbo estratégico que se pretende seguir, y desde siempre han constituido un reto para los investigadores debido a su naturaleza desestructurada y el nivel jerárquico en el que se encuentran alojadas (Ganga-Contreras, et al., 2018). Por otra parte, las decisiones estratégicas son responsabilidad de los directivos de una organización (Elbana, 2006), donde para ser eficaz en la toma de decisiones estratégicas, debe haber coherencia en los resultados de dichas decisiones (Mitchell, et al., 2011), y ello se refleja en el desempeño de las organizaciones (Ribeiro, et.al., 2016). Sin duda, cada vez es más frecuente tomar decisiones en escenarios con bastante incertidumbre (Riquelme-Castañeda, et.al., 2020, Calabretta, et al. 2017).

En el ámbito universitario, conceptualmente, la definición de decisión estratégica y sus principales características no distan demasiado de la presentadas en el párrafo anterior. Sin embargo, se debe relevar la mirada de consenso y participación en el proceso de toma de decisiones estratégicas (particularmente de las universidades que cuentan con financiamiento del Estado), debido a elementos culturales y estructuras corporativas particulares, donde se valoran los procesos que involucran a la comunidad interna y externa (Pedraja-Rejas y Rodríguez-Ponce, 2015). Por otra parte, las máximas autoridades universitarias (Juntas Directivas) quiénes toman decisiones estratégicas, debiesen facilitar la resolución de problemas que implica la gestión de las instituciones de educación superior, involucrando mejoras constantes que les permita adaptarse y enfrentar el complejo y cambiante entorno (Rojas y Bernasconi, 2009, Rodríguez, 2006).

\section{Gestión estratégica}

La gestión estratégica, considera intrínsecamente decisiones estratégicas, en la planeación e implementación. En otras palabras, delinean el quehacer de la organización, incorporando además el contexto en el cual se desenvuelve (Papadakis, et al., 2010; Elbana, et al., 2020). Por lo tanto, la gestión estratégica, no se debe simplificar definiéndola como sinónimo de planificación estratégica (Elbanna, 2006; Papadakis, et al., 2010), sino que es necesario considerar los procesos de definición, análisis, implementación y evaluación estratégica, esto es aplicable a cualquier tipo de organización (Pollanen, 2017).

El proceso de planificación, considera el análisis interno y externo (Ghonim, et al., 2020). En el análisis interno, se incorporan definiciones estratégicas, como la visión estratégica, misión, objetivos estratégicos, valores. En relación a la visión se refiere al camino que una organización toma con el fin de desarrollar y fortalecer sus actividades, expresando el curso estratégico de la organización en la preparación de su futuro (Porter, 1996) Por otra parte, la declaración de una misión, se posiciona en el presente describiendo cuál es el propósito de la existencia de una de una organización (Mainardes et al., 2014). Se consideran, por lo tanto, decisiones que proyectan una trayectoria, estableciendo objetivos de desempeño y eligiendo una estrategia capaz de producir los resultados deseados (Mainardes, et al., 2014). Por otra parte, previo a trazar el rumbo estratégico, se hace necesario conocer y analizar de manera profunda el entorno en el que se desenvuelve la organización (Porter, 2008). Este análisis tiene como objetivo revelar los impulsores de la competencia en un sector, de manera de identificar posibles oportunidades y amenazas en el ambiente externo y detectar fortalezas y debilidades al interior de una organización, para luego tomar decisiones que se encaminen al logro de ventajas competitivas (Mainardes et al., 2014). En este mismo sentido, según Papulova y Gazova (2016), "el papel del análisis estratégico se centra principalmente en examinar el efecto de factores críticos clave que provocan cambios en el medio ambiente y, en consecuencia, permiten anticipar cambios en el medio" p.575.

En relación al proceso de implementación, se debe considerar la importancia de lograr una alineación estratégica, la cual implica que en las organizaciones deba existir un equilibrio entre la estrategia y el contexto en que se desenvuelven, lo cual sin duda, tiene un impacto relevante en su desempeño (Ghonim, et al., 2020). En este proceso además juegan un rol relevante los liderazgos, para facilitar (o entorpecer) la implementación de las estrategias (Porter, 1996). Finalmente, el proceso de seguimiento y control,

En el ámbito de las universidades, Rojas y Bernasconi (2009), concuerdan que, "un buen gobierno universitario, necesita directrices claras, una estrategia de desarrollo institucional, una definición de misión y objetivos distintivos de una universidad" (p.213), todo lo anterior debe guiar las decisiones y alinear a la comunidad, tras una visión de futuro. Según Bennett (2002), quienes dirigen tiene un papel influyente en la formulación de políticas estratégicas y el establecimiento de parámetros en los que esa estrategia quiere ser lograda por la dirección de la universidad. En un contexto cada vez más competitivo, para lograr el éxito en la formulación e implementación de las estrategias, se requiere de decisiones asertivas, oportunas y orientadas hacia la visión de la organización. Por otra parte, Rodríguez, (2006), afirma que "la implementación de los procesos de dirección estratégica está en la toma de decisiones, allí se encuentra la fuente de ventaja competitiva" (p.55). Una ventaja competitiva, es una forma diferente adoptada por una empresa u organización, para desarrollar una actividad productiva o de servicio, en una posición superior a la de sus competidores. 
La propuesta de valor desarrollada es percibida por el mercado objetivo como un producto o servicio único, aumentando la fidelidad de los clientes o beneficiarios finales (Porter, 2008). Según Mainardes, et al., (2014), la esencia de la gestión estratégica consiste en desarrollar y retener una ventaja competitiva. Así, las organizaciones deben pensar cómo realizar su actividad, de una manera que sus rivales no lo pueden hacer o les sea difícil de imitar. Por otra parte, las dotaciones de recursos de las organizaciones determinan una ventaja competitiva sostenible, de allí la importancia de determinar las brechas de recursos para invertir en mejorar las potenciales debilidades (Porter, 2008). En lo concerniente a la gestión universitaria, si las organizaciones no logran diferenciarse y tener éxito, se debe a que sus recursos y talentos se diluyen y los competidores van captando ventajas. Lo anterior es especialmente importante para las universidades, por cuanto el expandir sus proyectos y desarrollar ventajas competitivas, dependerá de su capacidad de atraer talento superior (Riquelme-Castañeda, et al., 2020).

\section{Responsabilidad social universitaria (RSU)}

El concepto de RSU da cuenta de la contribución efectiva que las instituciones de educación superior hacen al desarrollo sustentable de la sociedad, a través de la gestión, formación educativa, investigación científica y la extensión universitaria (Gaete, 2016). Asimismo, dentro del contexto de la responsabilidad social, las universidades cumplen un rol fundamental, ya que éstas son las responsables de la creación y difusión del conocimiento, factor importante para promover el cambio social y el desarrollo económico (De Mesquita, et al., 2020), convirtiéndose así en instituciones estratégicas dentro de la sociedad. Según Martí y Martí-Vilar (2013), en los países latinoamericanos se ha materializado la responsabilidad social universitaria "desde la visión de una universidad con una importante faceta de trabajo con la comunidad mediante los programas de extensión universitaria y compromiso social, reflejando la importancia de concienciar al alumnado acerca de las diferentes realidades socioculturales de su entorno" p.148. De esta forma, el concepto de RSU podría plasmarse en un mayor interés por parte de las instituciones de educación superior, por renovar la forma de relacionarse con sus diferentes grupos de interés, para así responder a las demandas y necesidades comunitarias (Berghaeuser y Hoelsche, 2019; Barrios-Hernández y Olivero-Vega, 2020). En este sentido, en Chile las universidades son reconocidas por ser organizaciones que no persiguen fines de lucro, con un gran sentido de la vocación pública y de responsabilidad social, por lo que los directivos de éstas deben comprender la consigna de las instituciones de educación superior, de ser organismos que tienen la responsabilidad de crear una sociedad sostenible, considerando una amplia gama de grupos de interés (Larrán y Andrades-Peña, 2015).

\section{METODOLOGÍA}

La investigación apunta a responder cómo las decisiones estratégicas aportan al logro de ventajas competitivas, desde un enfoque de responsabilidad social universitaria. En este sentido, este artículo utiliza como metodología el estudio de caso, debido a su naturaleza, cuyo foco es la investigación de un fenómeno actual, dentro de un contexto real, (Yin, 1984). Esta metodología de estudio de caso cualitativo permite a los investigadores realizar una exploración en profundidad de fenómenos intrincados dentro de un contexto específico (Rashid, et al., 2019). Además, se consideró que era una metodología adecuada para aprender de la realidad de una situación estratégica a nivel directivo, considerando el entorno donde se desarrolla el fenómeno, tal como lo menciona, (Ramirez-Sánchez, et al., 2019). Se utilizó principalmente información secundaria e incluye una revisión teórica y análisis de documentos de trabajo e informes oficiales relacionados con el proyecto Becas Maule e información de prensa.

\section{CASO BECAS MAULE}

La Universidad Católica del Maule, es la primera universidad en la región del Maule en abrir la carrera de Medicina el año 2004, la cual nace con el propósito de asumir el desafío de "formar profesionales para satisfacer las necesidades de sus semejantes en materias de salud...y contribuir a lograr condiciones de mayor equidad y justicia social" (Consejo Superior en su Sesión Ordinaria № 6/2000). Con esta impronta es que se busca avanzar más allá del pregrado, para dar soluciones más complejas a las necesidades que había en el Maule, específicamente de profesionales médicos especialistas en áreas críticas de salud. En este momento, la UCM visualiza este escenario, siendo la única escuela de Medicina que tiene egresados en la región.

El caso de las Becas Maule se remonta al año 2012, cuando la Facultad de Medicina de la Universidad Católica del Maule (UCM), toma la decisión estratégica de ocuparse de la profunda brecha que existe en relación a la falta de médicos especialistas (profesionales médicos que han tenido una formación de a lo menos tres años (posterior al pregrado) y que han sido titulados por una universidad o certificados por una entidad reconocida) que existe en la región del Maule, área geográfica en la cual está inserta. En este contexto, la UCM diseñó un inédito programa de formación de cien médicos especialistas en y para la región de Maule, con el objetivo de reducir la brecha paciente/médico. Para ese entonces, en la región existía una 
de las mayores brechas de médicos especialistas en Chile, cerca de 6,0 profesionales por cada 10 mil habitantes, siendo la segunda tasa más baja del país. Las brechas de especialistas en la región del Maule alcanzaban 197 profesionales, de acuerdo a lo informado por el Servicio de Salud del Maule en julio 2012, siendo anestesiología, cirugía general, intensivista, tórax, traumatología, ginecología, medicina interna y neonatología, las mayores necesidades.

La UCM que, al momento de la implantación de este programa, aspiraba según su misión a "[...]la formación de profesionales integrales, con una visión crítica y constructiva, que guíen el desarrollo de las comunidades y organizaciones en las que se inserten [...]" (UCM, 2009, p.23), se propuso articular diferentes estrategias para la implementación del programa Becas Maule, formando alianzas estratégicas con el Gobierno Regional del Maule (GORE) y con el Servicio de Salud del Maule (SSM). Luego de siete años de vigencia ininterrumpida de este programa, la universidad ha logrado titular a 71 médicos especialistas, reduciendo en un $36 \%$ la brecha existente, con una inversión de 24 millones de dólares.

\section{Análisis estratégico del entorno e identificación del problema}

El Programa de Formación de Médicos Especialistas, representó para la UCM una oportunidad de contribuir a reducir la brecha de especialistas médicos en el sistema público de salud regional, colaborar en el cumplimiento oportuno de las exigencias legales de la Reforma Sanitaria, y posibilitar la dotación de especialistas médicos en hospitales de la red asistencial pública en ciudades importantes de la región, donde tradicionalmente era difícil la llegada de nuevos médicos con formación completa. Durante el análisis de pertinencia de este programa, la UCM enfatizó la necesidad de desarrollar capacidades que permanecieran en la región, como la atracción, formación y retención del recurso humano médico especialista. No era sencillo llevar adelante esta tarea, debido a múltiples factores, tales como la destrucción de la infraestructura hospitalaria a raíz del terremoto (magnitud de 8,8 grados Richter) ocurrido el año 2010, el cual deterioró significativamente la disponibilidad de recursos físicos y condiciones generales para el desempeño seguro y atractivo en los centros de salud. Por otra parte, en el marco del plan de reposición de la infraestructura, el gobierno contempló la construcción de un nuevo y moderno Hospital Regional, ubicado en la ciudad de Talca, el cual debía entrar en funcionamiento a mediados del año 2013. Este nuevo hospital demandaría una importante dotación de médicos especialistas inexistes en la región, con lo cual se corría el riesgo de contar un centro de salud sin capital humano competente para operarlo. El plan de reconstrucción incluyó hospitales para otras importantes ciudades de la región, tales como Curicó, Linares, Cauquenes, Constitución y Parral, los que debían entrar en funciones a partir del año 2014.

Respecto del desarrollo regional, el atractivo de una zona depende de la conectividad, acceso a colegios, universidades, cultura, condiciones ambientales, entre otros. Todos estos factores contribuyen al potencial atractivo para el profesional médico. En el caso particular de la región del Maule, ésta históricamente ha tenido un retraso en cuanto a la diversificación de su matriz productiva, centrada en actividades silvo agropecuarias, así como en sus índices de crecimiento e ingresos por persona inferiores al promedio nacional. Todo lo anterior, supuso un gran desafío para el desarrollo de este proyecto, tanto en el sentido de atracción de estudiantes para el programa de especialidades médicos, como el de contar con un claustro académico adecuado.

En cuanto a la calidad de red atención de salud pública de la región, para el año 2012, el panorama era muy desalentador, tanto por falta de infraestructura como por el éxodo de médicos especialistas post terremoto. Para esa fecha las listas de espera de atención crecían a diario y los pacientes debían esperar o ser trasladados a otras regiones del país. Es probablemente difícil que, en el sistema de salud de la región del Maule, se vuelvan conjugar las variables anteriormente descritas, de allí la importancia que la UCM haya sido capaz de mantenerse estratégicamente contactada con su realidad inmediata, y partir de ello lograr un buen punto de partida para desarrollar las estrategias necesarias que le permitieron capitalizar esta oportunidad.

\section{Formulación estratégica y análisis de capacidades internas}

Para el año 2012, la Facultad de Medicina de la UCM tenía tan solo tres años de existencia. No obstante, contaba con un claustro académico de calidad, motivado por mejorar las condiciones de salud en la región, aumentando las competencias de los profesionales del área. Además, la Facultad había definido en sus objetivos estratégicos, la necesidad de fortalecer sus programas de postgrado (PDE Facultad de Medicina, 2011), siendo además coherente con los valores de la Universidad, a saber, respeto por la persona humana, espíritu de servicio y responsabilidad social. En este sentido, el programa Becas Maule, se definió como una acción clave para el posicionamiento en términos misionales, de imagen y fortalecimiento de la Facultad.

La universidad estimó que la puesta en marcha e implementación del programa Becas Maule, tendría un costo aproximado de $\$ 12.470$ millones (recursos externos). Esta enorme necesidad de recursos económicos, supuso desde un principio, la necesidad de buscar alianzas estratégicas con partes interesadas (GORE y 
SSM). Se elaboró un detallado plan con los objetivos del programa de formación de especialistas, sus impactos para la población, plazo de ejecución y variables de control. Finalmente, se logró aunar las voluntades del GORE y SSM, aprobándose el total de recursos requeridos, desglosados de la siguiente manera: $\$ 6.410$ millones aportados por el GORE, $\$ 6.060$ millones por el SSM y $\$ 6.186$ millones, por parte de la UCM (valorizando la infraestructura y el recurso humano). Sin duda, esta alianza estratégica de trabajo colaborativo fue gravitante para el éxito del proyecto, constatando lo expuesto por Barrios-Hernández, et.als., (2020).

\section{Implementación}

La Universidad sentó las bases de su política de vinculación en el año 2004, la cual fue evolucionando y redefiniendo conceptos claves, que permitieron una mejor comprensión de las directrices. En este sentido el cambio de paradigma en la relación con su entorno, "pasando de una mirada asociada a la "Extensión" a otra que busca fortalecer una "Vinculación con el medio" efectiva desde una mirada horizontal y bidireccional de sus funciones" (Informe Autoevaluación UCM 2015-2020, p.176). Esta nueva mirada se condice con la propuesta e implementación del programa de Becas Maule, que es bidireccional, donde todas las partes se benefician, y además es horizontal, permitiendo el desarrollo del territorio y una mejora continua de sus procesos. Este programa se desarrolló entre los años 2013 y 2017, comenzó con trece especialidades y una subespecialidad y un total de veintisiete alumnos proyectados.

La Universidad Católica del Maule, a través de su Facultad de Medicina colocó a disposición del proyecto toda su infraestructura física tecnológica y la capacidad formadora, el cuerpo docente que reúne a la mayoría de los especialistas de la región del Maule y toda su red de apoyo, y que para la primera etapa contó con la colaboración de la Facultad de Medicina de la Pontificia Universidad Católica, supliendo el recurso humano especialista, que no estuviese disponible en el Maule. Es necesario comentar, que el recurso humano es un factor crítico de éxito en la implementación de las especialidades médicas, debido a que éstas tienen como particularidad la personalización del trabajo entre el becado y su tutor, requiriéndose el apoyo de un docente médico especialista de 11 horas por cada becado y 22 horas para el director de cada programa de especialidades, según las condiciones exigidas por la acreditación de dichos programas.

El programa de mejoramiento de la atención médica en especialidades para los usuarios de la Región del Maule, fue diseñado para ejecutarse en un plazo de cinco años y se inició impartiendo aquellas especialidades más deficitarias y de mayor demanda en la región del Maule, como eran: anestesia, medicina interna, cirugía, ginecología, obstetricia, pediatría y traumatología. La estrategia para mantener el recurso humano en la región, se enfocó principalmente en reclutar a los médicos jóvenes que ya trabajan en la región y atraer a quienes deseen instalarse en la región con un propósito de largo plazo, comprometiéndolos a quedarse en la región por a lo menos seis años posteriores a la finalización de la beca, a través de una plaza en la red asistencial y trabajo en los campos clínicos de la región.

\section{RESULTADOS}

El programa Becas Maule, surge desde la conexión de la universidad y sus académicos con su entorno. En este sentido, la relación desde sus inicios de la Facultad de Medicina con el Hospital Regional de Talca (el hospital con mayor complejidad de la región) y con los demás hospitales del Maule a través de sus campos clínicos, facilitó la detección de la imperiosa necesidad de médicos especialistas para la región. En este sentido, la Universidad da cuenta de que su vinculación con el medio es bidireccional, aportando no sólo con programas específicos para el Maule, sino también generando efectos positivos colaterales con la atracción de nuevos médicos, tanto los becarios como sus profesores tutores, quiénes prestan servicios a los hospitales de la región. Además de potenciar equipos de investigación dentro de la Universidad especialmente en áreas sensibles para la población, como es, por ejemplo, oncología.

Becas Maule, viene además a fortalecer el vínculo estratégico con los campos clínicos de la Universidad. Asimismo, se generan nuevas alianzas estratégicas en torno al programa, a saber, la relación de la triada que posibilita su implementación, Gobierno Regional del Maule, Servicio de Salud del Maule y Universidad Católica del Maule. En términos de la formación de los especialistas, la Universidad tiene desde la creación de la carrera de medicina un convenio de colaboración con la Pontificia Universidad Católica, el cual facilitó la formación de médicos en ciertos cursos o especialidades que no era posible entregar en Talca, debido a la complejidad o especificidad de la misma, lo cual hacía difícil contar con el recurso humano avanzado necesario. Finalmente, una alianza estratégica importante fue la que se comenzó a gestar fue con las otras casas de estudio, lo que permitió acceder a nuevos y mayores fondos para crear una segunda versión con mayor cantidad de becados y de especialidades.

Este proyecto logró desarrollar los primeros programas de posgrados en especialidades médicas de la región del Maule, sentando las bases para aumentar la producción científica en la región, potenciando estudios desde el territorio y focalizando la formación médica en las necesidades de la población. 
Finalmente, también es posible inferir que el posicionamiento de la Facultad de Medicina fue impactado positivamente por el programa. Hoy en día, la carrera de Medicina de la Universidad Católica del Maule, se ha posicionado como la carrera que, de acuerdo al ranking de Prueba de Selección Universitaria PSU, capta los mayores puntajes de ingreso en la región. Los años 2018-2019 y 2020, el primer seleccionado obtuvo los más altos puntajes de ingreso, 797, 798 y 795 puntos respectivamente, superando a la Universidad de Talca (estatal) que alcanzó 787, 783 y 769,45 puntos en el mismo periodo, y a la Universidad Autónoma (privada), según se publica en los sitios web de las universidades.

\section{DISCUSIÓN}

La conducción de la Facultad de Medicina de la UCM, da cuenta de una organización conectada con su entorno, conocedora en profundidad de los problemas y desafíos del sistema de salud pública y privada, lo cual le permitió capitalizar un importante conjunto de oportunidades antes que otras casas de estudios de la región del Maule, lo cual es coherente con lo planteado por Rodríguez, et al. (2018) y Elbana et al. (2020), al relevar como un elemento crítico el ambiente externo en la toma de decisiones estratégicas. Los médicos que son académicos en la UCM, también prestan servicios en los hospitales de la región, y tienen el conocimiento y la sensibilidad en relación con el sufrimiento de las personas más vulnerables que son quienes acuden a los recintos públicos, por tanto, el conocer a cabalidad el entorno, aumenta la consistencia de las decisiones estratégicas, como se demostró en el trabajo de Mitchell, et al. (2011). Asimismo, los médicos conocen el sello UCM, que realza los valores y la impronta a favor de quienes más lo necesitan, en palabras del rector de la época, la universidad debe "ser la oportunidad de desarrollo para la Región y sus habitantes en especial para los sectores menos favorecidos" (UCM, 2009). Respecto del sistema de enseñanza que se entregan en los programas de especialidades, los alumnos cuentan con cursos de ética médica o bioética, reforzando el carácter y los valores de la universidad, centrados en el respeto por las personas, espíritu de servicio y responsabilidad social.

En el proceso de decisiones estratégicas, del caso en estudio, concurrieron todos los estamentos de la universidad, desde la facultad, pasando por Rectoría y finalmente el Consejo Superior, confirmando una alineación estratégica, fundamentada no sólo en un pensamiento racional de éxito (necesario), sino en un pensamiento estratégico, que permite alinear a la organización en una visión estratégica compartida, como lo expone Papulova (2016). En esta fase, se destaca la calidad de la información disponible para la toma de decisiones, que jugó un rol muy relevante en relación a la asertividad y que es concordante, con lo expuesto por Ribeiro, et al. (2016), y el adecuado balance de riesgos que la universidad realizó del proyecto, lo cual la condujo a buscar alianzas estratégicas con partes interesadas.

La formulación de la estrategia fue solo el comienzo, posteriormente fue necesario contar con un buen proceso de gestión estratégica. Lo anterior se evidencia en el caso Becas Maule, en la claridad de los objetivos planteados, estrategias y planes de acción alineados, variables de control conocidas y claras, y una rendición de cuentas, sistemática y transparente. En relación a los objetivos claros, éstos permitieron enfocar las estrategias en la consecución de una meta "formar 100 médicos especialistas para el Maule". Esta declaración logró alinear los esfuerzos de todos quienes participaron en la formación de los especialistas, facilitando la implementación de los planes de acción. Respecto de la rendición de cuentas, en este caso, fue interna y externa. Internamente, exponiendo el avance del proyecto, en el contexto de su plan estratégico, tanto al Consejo de Facultad como al Consejo Superior de la Universidad, y externamente, rindiendo a las contrapartes del programa, el Gobierno Regional y el Servicio de Salud del Maule, quienes trabajaron con altos estándares de control de la calidad en la ejecución de este programa. Los aliados estratégicos, que aportan desde el inicio y se involucran, se sienten más comprometidos con el logro de los objetivos, esta fue otra de las claves para el éxito de la implementación del programa, la alianza estratégica con el GORE y SSM, la cual facilitó la gestión del proyecto, aportando no solo recursos, sino también, imponiendo más mecanismos de control y de rendición de cuentas (como se mencionó anteriormente), lo que aumentó la transparencia del proyecto, como expone Elbana (2006), las decisiones estratégicas, contienen componentes racionales y políticos que al ser comprendidos por los tomadores de decisiones facilitan las decisiones estratégicas, en todo el proceso (formulación, implementación y seguimiento). El proceso estratégico, que tan claramente se advierte en la descripción anterior, es resultado de una visión estratégica clara que permite focalizar esfuerzos, y alinear decisiones en pro de la consecución de los objetivos estratégicos.

La responsabilidad social universitaria, plasmada en la formulación e implementación de las Becas Maule, promueve sin duda el cambio social y desarrollo de la comunidad, como propone De Mesquita, et al.(2020), a través de la formación de capital humano avanzado, que además está en coherencia con los valores de la universidad, ya que está dando respuesta a una de las necesidades básicas de nuestra sociedad como es la salud de los más necesitados. Desde el punto de vista social y productivo, este programa permitió la dotación de recurso humano calificado en la operación y equipamiento de los nuevos centros asistenciales que se estaban construyendo y construirán en las ciudades de Talca, Curicó, Linares, Cauquenes, Constitución y Parral, abriendo para la Facultad Medicina, un valioso número de campos clínicos. Además, este programa, 
dio inicio a un círculo virtuoso de calidad: más especialistas trabajando en equipo, mejores hospitales, mejores condiciones de trabajo y por lo tanto lugares atractivos para el desempeño laboral. Todo ello también ha tenido un impacto positivo en los grupos de interés de la universidad, ganando una importante valoración frente al GORE y SSM, instituciones que creyeron y apostaron por la Facultad de Medicina de la UCM. Son estos aliados estratégicos, parte de los grupos de interés de la universidad, quiénes aportaron recursos para implementar este proyecto Becas Maule, y respaldaron esta dimensión social, que es parte de su misión como universidad, como lo exponen, Berghaeuser y Horlsche, (2019).

Los especialistas médicos formados en UCM y con destino en la región del Maule, han posibilitado un funcionamiento eficiente de la red asistencial pública. De estos médicos especialistas, surgirán naturalmente los nuevos líderes en el área de salud de la región, ellos llevan la impronta y el alma máter de la UCM. Otras externalidades positivas derivadas del programa son, la composición de equipos profesionales y docentes calificados en la UCM, lo que se ha traducido en aumentar la investigación en el ámbito médico de relevancia para la región, impactando directamente en las políticas públicas de salud locales.

Finalmente, podemos afirmar que el proceso de decisión e implementación estratégica, llevado adelante por la Facultad de Medicina de la UCM en el marco del programa Becas Maule, no solo ha contribuido a cumplir con el rol social que a la universidad le compete, sino también ha sido una importante fuente de generación de ventajas competitivas para la carrera de Medicina de la UCM, permitiéndole captar a los mejores puntajes de ingreso; aumentar los índices de producción científica, acceder a un extenso conjunto de campus clínicos, y formar los futuros profesionales que liderarán el sistema de salud público privado de la región del Maule.

\section{CONCLUSIONES}

El proyecto Becas Maule ha sido considerado exitoso, desde el punto de vista de la asertividad y oportunidad de las decisiones estratégicas plasmadas en un proyecto que en su esencia internaliza la responsabilidad social universitaria, y es capaz de reunir a grupos interesados de la Universidad Católica del Maule, para lograr una ventaja competitiva sostenible. De acuerdo a los resultados y análisis, se pueden extraer las siguientes conclusiones: (1) importancia de la conexión con el entorno por parte de la Universidad para capitalizar oportunidades de forma temprana, (2) se debe relevar la calidad y oportunidad de la información para la toma de decisiones, que permitió lograr alianzas estratégicas con las partes interesadas, (3) la alineación estratégica al interior de la UCM como factor clave para el éxito del proceso de planeación e implementación de las Becas Maule. Sin duda, todos estos elementos convergieron para lograr no sólo la formación de nuevos médicos para la región, sino su permanencia, siendo esta la base para el logro de ventajas competitivas de la UCM en el área de medicina en el Maule. Las Becas Maule, son un excelente ejemplo de cómo una universidad regional, a pesar de las limitaciones en cuanto a tamaño, capacidades y recursos financieros, fue capaz de cumplir con su rol de ser una universidad socialmente responsable, contribuyendo de forma efectiva al desarrollo sustentable de la sociedad, a través de la gestión estratégica y formación educativa.

\section{REFERENCIAS}

Barrios-Hernández K., y Olivero-Vega, E., Relación universidad-empresa-estado. Un análisis desde las instituciones de educación superior de Barranquilla-Colombia, para el desarrollo de su capacidad de innovación, http://dx.doi.org/10.4067/S0718-50062020000200021, Formación Universitaria, 13(2), 21-28 (2020)

Bennett, B., The New Style Boarsd of Governors - Are they working?, Higher Education Quarterly, 56(3), 287-302 (2002)

Berghaeuser, H., y Hoelsche, M., Reinventing the third mission of higher education in Germany: political frameworks and universities' reactions, https://doi.org/10.1007/s11233-019-09030-3, Tertiary Education and Management, 26, 57-76 (2019)

Calabretta, G., Gemser, G., y Wijnberg, N., The Interplay between intuition and rationality in strategic decision making: a paradox perspective, http://dx.doi.org/10.1177/0170840616655483 ,Organization Studies,38(3-4), 365-401 (2017)

De Mesquita, R.F., Intravaia, D., y otros 3 autores, Disclosure of social responsability and performance of Brazilian universities, https://doi.org/10.25112/rgd.v17i1.2022, Gestao e Desenvolvimento, 17(1), 70-86 (2020)

Elbana, S., Strategic decision-making: process perspectives, International Journal of Management Reviews, 8(1), 1-20 (2006)

Elbana, S., Thanos, I., y Jansen, R., A., Literature review of the strategic decision-making context: a synthesis of previous mixed findings and an agenda for the way forward, https://doi.org/10.3917/mana.232.0042, M@n@gement, 23(2), 42-60 (2020)

Gaete, R., La universidad y la responsabilidad social, REXE-Revista de Estudios y Experiencias en Educación, 5(10), 129-145 (2016)

Ganga-Contreras, F., Durán-Seguel, I., y Rodríguez-Ponce, E., Racionalidad, como punto focal de las decisiones estratégicas: un acercamiento teorico conceptual, Revista Espacios, 39(26), 5-15 (2018) 
Ghonim, M., Khashaba, N., Al-Najaar, H., y Khashan, M., Strategic alignment and its impact on decision effectiveness: a comprehensive model, https://doi.org/10.1108/IJOEM-04-2020-0364, International Journal of Emerging Markets, 1-22, (2020)

Halibas, A., Sibayan, R., y Maata, L., The penta helix model of innovation in Oman: an HEl perspective, Interdisciplinary Journal of Information, Knowledge, and Management, 12, 159-172 (2017)

Larrán, M., y Andrades-Peña, F., Análisis de la responsabilidad social universitaria desde diferentes enfoques teóricos, Revista Iberoamericana de Educación Superior (RIES), VI(15), 91-107 (2015)

Mainardes, E.W., Ferreira, J.J., y Raposo, M.L., Strategy and strategic management concepts: are they recognised by management students?, https://doi.org/10.15240/tul/001/2014-1-004 , E\&M Ekonomie a Management, 17(1), 43-61 (2014)

Martí, J., y Martí-Vilar, M., Una década de responsabilidad social universitaria en iberoamérica, Revista Española del Tercer Sector, 25, 145-162 (2013)

Mitchell, J.R., Shepherd, D., y Sharfman, M., Erratic strategic decisions: when and why managers are inconsistent in strategic decision making, Strategic Management Journal, 32(7), 683-704 (2011)

Papadakis, V., Thanos, I., y Barwise, P., Research on strategic decisions: taking stock and looking ahead, en Handbook of decision making, John Wiley \& Sons, 31-69, Chichester, Inglaterra, (2010)

Papulova, Z., y Gazova, A., Role of strategic analysis in strategic decision-making, https://doi.org/10.1016/S22125671(16)30301-X, Procedia Economics and Finance, 39, 571-579 (2016)

Pedraja-Rejas, L., y Rodríguez-Ponce, E., Dirección estratégica y gestión en instituciones de educación superior, en La educación superior en Chile: transformación, desarrollo y crisis, Ediciones UC, 475-515, Santiago, Chile, (2015)

Pollanen, R., Abdel-Maksoud, A., Elbanna, S., y Mahama, H., Relationships between strategic performance measures, strategic decision-making, and organizational performance: empirical evidence from Canadian public organizations, https://doi.org/10.1080/14719037.2016.1203013, Public Management Review, 19(5), 725-746 (2017)

Porter, M.E., The five competitive forces that shape strategy, Harvard Business Review, 86(1), 78-93 (2008)

Porter, M.E., What is the strategy?, Harvard Business Review, 74(6), 61-78 (1996)

Ramirez-Sánchez, M., Rivas-Trujillo, E., y Cardona-Londoño, C., El estudio de caso como estrategia metodológica, Revista Espacios, 40(23), 1-8 (2019)

Rashid, Y., Rashid, A., y otros 3 autores, Case study method: a step-by-step guide for business researchers, https://doi.org/10.1177/1609406919862424, International Journal of Qualitative Methods (18), 1-13 (2019)

Ribeiro, I., Ribeiro, F., Portugal, M., y Paraiso De Campos, B., Relações teóricas e conceituais em tomada de decisão estratégica, Revista Eletrônica de Estratégia \& Negócios, 9(2), 57-84 (2016)

Riquelme-Castañeda, J.A., Pedraja-Rejas, L.M., y Vega-Massó, R.A., El liderazgo y la gestión en la solución de problemas perversos. Una revisión de la literatura, http://dx.doi.org/10.4067/S0718-50062020000100135, Formación Universitaria, 13(1), 135-144 (2020)

Rodríguez, E., El proceso de toma de decisiones en las universidades públicas, Calidad en la Educación, 24, 49-63 (2006)

Rodríguez-Cruz, Y., y Pinto, M., Modelo de uso de información para la toma de decisiones estratégicas en organizaciones de información, https://doi.org/10.1590/2318-08892018000100005, Transinformação, 30(1), 51-64 (2018)

Rojas, A., y Bernasconi, A., El gobierno de las universidades en tiempo de cambio, en Desafíos y perspectivas de la dirección estratégica de las instituciones universitarias, Ed. CNA-Chile, 183-214, Santiago, Chile (2009)

UCM, Plan de desarrollo Estratégico-UCM 2009-2013, Ed. Universidad Católica del Maule, Talca, Chile (2009)

Yin, R., Case Study research: design and methods. Ed. Sage Publications, California, USA (1984) 